\title{
Quinze ans après le SRAS : Principaux jalons de I'intervention en cas d'urgence touchant la santé publique du Canada
}

\author{
$\mathrm{T} \mathrm{Tam}^{1, \dagger *}$
}

\section{Résumé}

Cette année marque le $15^{\mathrm{e}}$ anniversaire du syndrome respiratoire aigu sévère (SRAS) au Canada et le $100^{\circ}$ anniversaire de l'épidémie de grippe espagnole de 1918. Ces événements, au même titre que d'autres événements récents touchant la santé publique nous offrent l'occasion de revoir la situation sur le plan de l'intervention en cas d'urgence touchant la santé publique du Canada et l'évolution de celle-ci, au cours des quinze dernières années - depuis le SRAS à la grippe pandémique $\mathrm{H} 1 \mathrm{~N} 1$ de 2009 et aux maladies à virus Ebola et à virus Zika. Des enseignements clés ont été tirés et des jalons ont été atteints : ceux-ci ont façonné et amélioré notre approche et nos structures en matière d'intervention. Bien que le SRAS nous ait fait prendre conscience de la nécessité de renforcer notre capacité en matière de surveillance et de prévention des infections dans des contextes de soins de santé et ait mené à la formation de I'Agence de la santé publique du Canada (ASPC), il a également renforcé notre gouvernance fédérale/provinciale/territoriale (FPT) au plus haut niveau et mené à la conclusion d'ententes au chapitre de l'aide mutuelle pancanadienne et de l'échange de renseignements sur les maladies infectieuses. Notre capacité collective au chapitre des laboratoires de santé publique a également été renforcée par une intervention constante et par la mise en commun de recherches et de diagnostics avancés. À l'avenir, il importera de se pencher sur la conception de stratégies et de structures évolutives ou modulaires en matière d'intervention en cas d'urgence qui sont appropriées sur le plan socioculturel et qui font appel à une communication sur les risques stratégique fondée sur des données probantes qui demeurent toujours essentielles, tout particulièrement à la lumière de la quantité de fausses informations et de la vitesse à laquelle celles-ci se répandent. Au vu de la réalité mondiale actuelle, nous devons reconnaître que toute menace sur le plan de la santé publique qui demeure non maîtrisée n'importe où dans le monde pose le risque de devenir très rapidement une menace pour la santé publique au Canada. Nous devons élaborer, maintenir et partager nos meilleures pratiques en matière de santé publique à l'échelle mondiale car, si nous négligeons d'agir en ce sens, ce sera à nos risques et périls.
Affiliations

${ }^{1}$ Agence de la santé publique du Canada, Ottawa (Ontario)

† Note : La Dre Tam est l'administratrice en chef de la santé publique du Canada

*Correspondance : drtheresa. tam@canada.ca

Citation proposée : Tam T. Quinze ans après le SRAS : Principaux jalons de l'intervention en cas d'urgence touchant la santé publique du Canada. Relevé des maladies transmissibles au Canada. 2018;44(5):109-12. https://doi.org/10.14745/ccdr.v44i05a01f

Mots clés : Intervention en cas d'urgence touchant la santé publique, SRAS : syndrome respiratoire aigu sévère, grippe, grippe pandémique, grippe espagnole de 1918, Ebola, Zika

\section{Introduction}

En 2018, nous célébrons à la fois le $100^{\mathrm{e}}$ anniversaire de la pandémie de grippe espagnole et le $15^{\mathrm{e}}$ anniversaire $d u$ syndrome respiratoire aigu sévère (SRAS) au Canada. S'offre ainsi à nous une excellente occasion de réfléchir sur l'expérience du Canada en matière de préparation et d'intervention en cas d'urgence touchant la santé publique au cours des récentes décennies.

Les urgences en matière de santé publique sont des événements complexes, de grande envergure, qui doivent donner lieu à une intervention qui s'appuie sur une participation complète du système de santé de même que sur la mobilisation d'une multitude de secteurs et/ou de l'ensemble de la société. Au-delà des nombreuses rencontres individuelles qui visent à prodiguer des soins aux patients dans une multiplicité de contextes de soins de santé, s'impose la nécessité de se doter d'une approche axée sur la population afin de gérer les défis fréquemment exceptionnels sur le plan du triage et du traitement en plus d'assurer la gestion du suivi, de la propagation au sein de la population et des impacts plus larges sur la société. Cette approche s'articule autour de nombreuses composantes dont la gouvernance, la surveillance, le diagnostic, l'identification et l'évaluation des risques, les mesures de santé publique (hygiène, éloignement social), les interventions spécifiques (vaccins et 
médicaments), le contrôle et la prévention des infections, la gestion clinique, les opérations et les communications. En découle la nécessité de se doter d'une approche en matière d'intervention qui fait intervenir de multiples secteurs face à de telles urgences, qui prévoit notamment la participation des responsables des services sociaux, des collectivités locales et des services non gouvernementaux.

Le présent commentaire a pour objectif de résumer les expériences et les principaux jalons du Canada s'agissant de faire évoluer notre capacité nationale au chapitre des mesures et des interventions en cas d'urgence touchant la santé publique au cours des quinze dernières années, en plus de relever les tendances et les défis actuels.

\section{Expérience du Canada}

Le SRAS a pris le monde entier par surprise en 2003 et bien que cette épidémie eût été comparativement circonscrite en termes de nombre total de cas, elle a néanmoins posé un formidable défi sur le plan de la santé publique, au Canada. Au cours d'une relativement brève période de cinq mois furent signalés 438 cas probables et soupçonnés de SRAS, y compris 44 décès (1). La plupart de ces cas étaient liés à des événements de transmission nosocomiale qui, eux-mêmes, ont entraîné 100 cas et trois décès, parmi les travailleurs de la santé. Le SRAS nous a donc fait prendre conscience de la nécessité de renforcer notre capacité en matière de surveillance et de prévention des infections dans des contextes de soins de santé. Plusieurs milliers d'autres personnes ont vu leur existence perturbée puisqu'elles ont été invitées à se mettre elles-mêmes en quarantaine afin d'éviter que la maladie ne se transmette ou ont été tenues de le faire.

Bien que le risque de propagation pandémique du nouveau coronavirus du SRAS, auquel étaient associées une morbidité et une mortalité beaucoup plus élevées, ne se soit finalement pas matérialisé, nombreuses ont été les difficultés liées au fait de contenir cette nouvelle maladie infectieuse et de réagir à celle-ci. Ces défis et les enseignements connexes qui en ont été tirés ont été décrits de manière détaillée dans le document intitulé Leçons de la crise du SRAS - Renouvellement de la santé publique au Canada - rapport du Comité consultatif national sur le SRAS et la Santé publique (1). Parmi ceux-ci figurent notamment la nécessité $d$ 'assurer une meilleure coordination des mesures d'intervention adoptées par les multiples ordres de gouvernement et la nécessité d'une reconnaissance accrue ou renouvelée de la menace pour la santé publique que posent les maladies infectieuses émergentes ou réémergentes. Bien que l'émergence du SRAS n'ait pas été prédite, une pandémie de grippe était et demeure toujours considérée comme inévitable dans le contexte des mesures d'urgence touchant la santé publique. En cela, dans le but de reconnaître et de partager largement et officiellement la planification fédérale/provinciale/territoriale (FPT) coordonnée, fut publié, pour la première fois, en 2004, le Plan canadien de lutte contre la pandémie d'influenza (PCLPI), lequel intégrait les enseignements tirés du SRAS. Grâce à une version mise à jour et constamment réactualisée du Plan canadien de lutte contre la pandémie d'influenza, publié en 2006, le Canada était beaucoup mieux préparé à répondre à l'émergence, à la propagation et à la pandémie mondiale du nouveau virus de la grippe H1N1 de 2009 provenant du Mexique, qui s'en est suivie (2). Le Canada a profité d'importants investissements en matière de renforcement des capacités comme en s'assurant $d^{\prime}$ un accès à des stocks d'antiviraux et à son propre vaccin contre la pandémie. Dans les faits, la pandémie H1N1 de 2009 n'a pas été aussi grave que celle imputable à certains de ses prédécesseurs. Par conséquent, durant et après la pandémie H1N1, les gouvernements nationaux et l'Organisation mondiale de la santé (OMS) ont reconnu qu'il y avait lieu de se doter d'une réponse plus souple et plus adaptable à l'égard des futures pandémies pour tenir compte des situations locales et régionales (2). L'épidémie de maladie à virus Ebola qui a débuté en Afrique occidentale, vers la fin de 2013, portait non seulement sur une maladie grave présentant des taux de morbidité et de mortalité élevés, mais elle a également suscité une épidémie de peur. Elle a démontré les graves répercussions d'une approche déficiente sur le plan des communications de même que l'importance que revêt le fait de tenir compte des croyances culturelles locales et $d$ 'assurer un leadership local au niveau de l'intervention. Cette épidémie représente également un exemple poignant de la nécessité de renforcer les capacités en matière d'intervention d'urgence touchant la santé publique dans chaque pays et de mieux coordonner les capacités d'intervention mondiale rapide, y compris sur le plan de la recherche, pour que nous soyons en mesure de protéger tant autrui que nous-mêmes $(3,4)$.

Un autre exemple du caractère variable et imprévu des événements de maladies nouvelles concerne l'épidémie de maladie à virus Zika qui a touché les Amériques. Le virus Zika a été déclaré une urgence de santé publique de portée internationale par l'OMS en février 2016. II convient de souligner que cette déclaration ne reposait pas sur les éléments qui étaient alors connus au sujet de l'infection à virus Zika (5). Elle reposait plutôt sur ce qui n'était pas connu au sujet des groupes de microcéphalie, du syndrome de Guillain-Barré (SGB) et d'autres anomalies neurologiques associées dans le temps et selon le lieu aux épidémies d'infection à virus Zika signalées depuis le Brésil et, de façon rétrospective, depuis la Polynésie française (6). Cette approche marquée au sceau de la précaution et de l'anticipation s'est avérée essentielle pour stimuler la réponse internationale en vue de la réalisation d'études et de la surveillance de même que des recherches rapides qui s'avéraient nécessaires pour étayer une réponse efficace en matière de santé publique.

Le Canada est actuellement en voie de répondre à la crise de santé publique consécutive à une épidémie de ce qui sembleraient être des surdoses et des décès apparemment liés à la consommation d'opioïdes (7). Jusqu'à présent, cette crise a entraîné le décès de près de 3000 Canadiens en 2016 alors que l'on prévoit 4000 décès ou plus, en 2017 et que la situation continue d'évoluer (8). Plusieurs de nos stratégies et de nos structures en matière d'intervention d'urgence élaborées, à l'origine, pour faire face aux urgences visant les maladies infectieuses, ont été adaptées pour faire face à cette crise de santé publique d'origine non infectieuse. Les besoins associés à la crise actuelle des opioïdes nous ont permis de réexaminer ce en quoi constitue une urgence touchant la santé publique et la meilleure façon d'intervenir à l'égard des défis de santé publique de nature non infectieuse. 


\section{Principaux jalons}

Depuis le SRAS, le Canada a accompli des gains importants à l'égard de sa capacité de répondre efficacement aux défis de santé publique que représentent les graves épidémies de maladies infectieuses. Suite aux recommandations du Comité consultatif national sur le SRAS et la Santé publique, un certain nombre d'éléments fondamentaux d'une intervention en cas d'urgence touchant la santé publique ont été mis en place ou améliorés. Parmi ceux-ci figure la création de l'Agence de la santé publique du Canada et l'établissement d'un Conseil du Réseau de santé publique (RSP) FPT à titre de forum de collaboration, de coordination et de gouvernance. Le RSP, au même titre que le Conseil des médecins hygiénistes en chef peut rapidement former un comité consultatif spécial (CCS) pour coordonner et gérer les événements ou les urgences nationales touchant la santé publique. De tels comités consultatifs spéciaux ont été mis sur pied lors de la grippe pandémique de 2009, lors de l'épidémie à virus Ebola survenue en Afrique occidentale et, plus récemment, en 2016, pour faire face à l'épidémie actuelle de décès liés aux opioïdes.

En 2009, conscients du fait que les gouvernements FPT étaient dotés de capacités variables sur le plan de la santé publique et que la collaboration s'avérait bénéfique lorsqu'une administration pouvait se retrouver débordée face à une urgence ou à une crise de santé publique, les ministres de la Santé ont signé le Protocole d'entente sur la prestation d'une aide mutuelle en rapport avec les ressources en santé lors d'une situation d'urgence mettant en cause la santé publique (PE sur I'aide mutuelle). Un Cadre de travail opérationnel pour des demandes d'aide mutuelle (CTODAM) pour les professionnels de la santé a depuis lors été élaboré afin de mettre en pratique les principes clés décrits dans le protocole d'entente, l'Agence de la santé publique du Canada (ASPC) agissant à titre de centre de coordination. Un exemple de l'utilité d'un tel mécanisme tient à sa mise en application lors des inondations survenues en Alberta, en 2013, alors que plusieurs administrations ont contribué de l'expertise et des spécialistes en santé de l'environnement pour appuyer les efforts de rétablissement consécutif aux inondations. Pour rehausser la capacité conjointe des gouvernements FPT, ceux-ci travaillent ensemble à la mise en place de centres de soins collaboratifs pour faire face à la maladie à virus Ebola à travers le pays afin que puissent être fournis des soins spécialisés en présence d'agents pathogènes à haut niveau de confinement.

La surveillance et l'échange rapides de renseignements s'avèrent essentiels à toute intervention efficace en matière de santé publique. En 2016, les ministres FPT de la Santé ont signé I'Entente multilatérale sur l'échange de renseignements (EMER), en vue de l'échange de renseignements relatifs à la surveillance des maladies infectieuses et à la gestion des événements de santé publique pancanadiens ou touchant plusieurs administrations, et les urgences de santé publique de portée internationale. Bien qu'il puisse demeurer délicat d'obtenir des renseignements cohérents au sein d'un système fédéré dont les administrations disposent de capacités variables, I'EMER représente une étape significative sur la voie de l'officialisation de l'échange de renseignements lors d'urgences mettant en cause des maladies infectieuses.
Les progrès technologiques signifient que la capacité en matière de diagnostic des laboratoires du Canada a évolué au cours des quinze dernières années. La génomique et d'autres techniques moléculaires fournissent désormais des éléments probants tout à fait détaillés lors des études en sécurité publique, y compris dans le cas des éclosions de maladies d'origine alimentaire. Le Réseau des laboratoires de santé publique du Canada (RLSPC) est un réseau de laboratoires de santé publique fédéraux et provinciaux qui est devenu un mécanisme bien établi pour collaborer de manière efficace au renforcement des capacités des laboratoires et de l'intervention face aux nouvelles menaces comme celles que représentent les maladies à virus Zika et Ebola. Le Laboratoire national de microbiologie (LNM) est parvenu à développer rapidement des tests de diagnostic pour de nouveaux pathogènes, comme le virus à Zika, lesquels pourraient être requis pendant une période de temps relativement longue avant que toutes les administrations soient en mesure d'accéder à des tests dûment validés, disponibles sur le commerce pour effectuer leurs propres tests.

Un autre accomplissement important tient aux recherches fondamentales qui ont été réalisées avec succès au LNM de I'ASPC, lesquelles ont éventuellement mené à l'élaboration et à la mise en œuvre d'un vaccin efficace pour l'Ebola et à un médicament composé d'anticorps monoclonaux (ZMapp) en collaboration avec le secteur privé, des organismes nationaux de financement de la recherche, des gouvernements et des chercheurs internationaux. Cette réalisation nous rappelle l'importance que revêtent les préparatifs sur le plan de la recherche, y compris la mise en place de réseaux de recherche, comme le Réseau canadien de recherche sur l'immunisation, qui peuvent être immédiatement mis en œuvre lors d'une intervention (9).

\section{Tendances actuelles}

Dans l'avenir, il importera d'envisager la conception de stratégies et de structures d'intervention d'urgence plus adaptables ou modulaires afin que l'on puisse réaliser des interventions plus souples face à n'importe quelle menace touchant la santé publique. À titre d'exemple, la version mise à jour du document Préparation du Canada en cas de grippe pandémique : Guide de planification pour le secteur de la santé (PCGP), publié en 2015, inclut quatre scénarios de planification possibles pour illustrer l'importance de dresser des plans et des stratégies d'intervention qui soient flexibles et adaptables aux circonstances (2). Le PCGP présente également des éléments déclencheurs d'intervention qui reposent sur l'émergence de nouveaux virus et l'activité pandémique au Canada.

En octobre 2017, ayant pris conscience du fait qu'il s'avérait impossible de se doter d'un plan de préparation adapté à une maladie en particulier pour chacun des pathogènes pouvant survenir lors d'une urgence ou d'un événement touchant la santé publique, le RSP approuvait un Plan d'intervention de la santé publique fédérale, provinciale et territoriale face à des menaces biologiques (10). Le plan d'intervention décrit les rôles, les responsabilités et les pouvoirs des gouvernements FPT en ce qui a trait à la santé publique et à la gestion des urgences, un concept opérationnel établissant quatre niveaux de réponse évolutifs et une structure de gouvernance qui coordonne 
I'intervention entre les territoires de compétence. Le plan facilite également la mobilisation efficace parmi les responsables de la gestion des urgences en matière de santé, de la prestation des soins de santé et de la santé publique.

Une bonne approche stratégique en matière de communication sur les risques continuera d'être un élément essentiel en plus de représenter un défi perpétuel dans un contexte d'intervention d'urgence, tout particulièrement au vu de la vaste quantité de fausses informations et de la facilité avec laquelle celles-ci parviennent à la population canadienne. Pour lutter contre les épidémies de peur, les établissements et les responsables de la santé publique doivent continuer d'incarner la voie la plus crédible, en période d'incertitude.

Nous devons nous doter d'interventions et d'une planification en matière de santé publique appropriées sur le plan socioculturel qui tient compte des Premières Nations, des Inuit, des Métis et des autres groupes de notre population canadienne diversifiée. Alors que nous gardons en mémoire les répercussions particulièrement dévastatrices de la grippe espagnole de 1918 sur les populations autochtones, la constante nécessité de collaborer avec les collectivités et les organisations autochtones à la planification et à la préparation face aux urgences touchant la santé publique et aux interventions à l'égard de celles-ci continue de s'imposer à nous.

\section{Conclusion}

Bien que nous ne puissions jamais être trop préparés, et que des travaux continus s'imposent encore, la situation s'est considérablement améliorée sur le plan de la préparation et de la capacité d'intervention en cas d'urgence touchant la santé publique du Canada au cours des quinze dernières années. Ces activités ont débuté avec l'intervention face au SRAS, et se sont développées avec chaque urgence de santé publique successive survenue depuis lors. Les récentes décennies ont été caractérisées par l'émergence et la propagation de maladies infectieuses, à travers le monde, qui appellent au renforcement de notre capacité d'intervention mondiale (11).

Au vu de la réalité mondiale actuelle, nous devons reconnaître que toute menace sur le plan de la santé publique qui demeure non maîtrisée n'importe où dans le monde pose le risque de devenir très rapidement une menace pour la santé publique au Canada. Le soutien que nous offrons à d'autres pays, pour rehausser la capacité mondiale de détecter, de rendre compte, de confiner et de traiter les menaces pour la santé publique nous permet également de former des partenariats internationaux essentiels et $d$ 'acquérir des connaissances en matière d'intervention tout en protégeant activement nos propres meilleurs intérêts.

Tous les enseignements que nous avons tirés de la crise du SRAS, et au cours des quinze années qui ont suivies nous montrent que nous devons élaborer, maintenir et partager nos meilleures pratiques en matière de santé publique car, si nous négligeons d'agir en ce sens, ce sera à nos risques et périls.

\section{Références}

1. Leçons de la crise du SRAS - Renouvellement de la santé publique au Canada - rapport du Comité consultatif national sur le SRAS et la Santé publique. Ottawa $(\mathrm{ON})$ : Santé Canada; 2004. https://www.canada.ca/fr/sante-publique/services/ rapports-publications/lecons-crise-sras-renouvellement-santepublique-canada.html

2. Plans d'intervention en cas de pandémie. Préparation du Canada en cas de grippe pandémique : Guide de planification pour le secteur de la santé. Ottawa (ON): Agence de la santé publique du Canada; 2015. https://www.canada.ca/fr/santepublique/services/grippe-influenza/preparation-canada-casgrippe-pandemique-guide-planification-secteur-sante.html

3. Organisation mondiale de la Santé. Rapport du Groupe d'experts chargé de l'évaluation intérimaire de la riposte à Ebola. http://www.who.int/csr/resources/publications/ebola/ ebola-panel-report/fr/

4. Moon S, Sridhar D, Pate MA, Jha AK, Clinton C, Delaunay S, Edwin V, Fallah M, Fidler DP, Garrett L, Goosby E, Gostin LO, Heymann DL, Lee K, Leung GM, Morrison JS, Saavedra J, Tanner M, Leigh JA, Hawkins B, Woskie LR, Piot P. Will Ebola change the game? Ten essential reforms before the next pandemic. The report of the Harvard-LSHTM Independent Panel on the Global Response to Ebola. Lancet 2015 Nov;386(10009):2204-21. http://dx.doi.org/10.1016/S01406736(15)00946-0

5. Organisation mondiale de la Santé. Déclaration sur la 1 ère réunion du Comité d'urgence du Règlement sanitaire international concernant le virus Zika. Février 2016. http:// www.who.int/mediacentre/news/statements/2016/1stemergency-committee-zika/fr/

6. Heymann DL, Hodgson A, Sall AA, Freedman DO, Staples JE, Althabe F, Baruah K, Mahmud G, Kandun N, Vasconcelos PF, Bino S, Menon KU. Zika virus and microcephaly: why is this situation a PHEIC? Lancet 2016 Feb;387(10020):719-21. http://dx.doi.org/10.1016/S0140-6736(16)00320-2

7. Santé Canada. Ce qu'il faut savoir sur la crise des opioïdes au Canada. Santé Canada; Ottawa (ON): 22 mars, 2018, https:// www.canada.ca/fr/services/sante/campagnes/preventiontoxicomanie.html

8. Santé Canada. Décès apparemment liés à la consommation d'opioïdes [consulté le 27 mars 2018]. Ottawa (ON); Santé Canada; 2018. https://www.canada.ca/fr/sante-canada/ services/toxicomanie/abus-medicaments-ordonnance/ opioides/deces-lies-opioides.html

9. Réseau canadien de recherche sur l'immunisation (RCRI). http://cirnetwork.ca/

10. McNeill R, Topping J. Plan d'intervention de la santé publique fédérale, provinciale et territoriale face à des menaces biologiques. Relevé des maladies transmissibles au Canada. 2018;44(1):1-6. https://www.canada.ca/fr/sante-publique/ services/rapports-publications/releve-maladies-transmissiblescanada-rmtc/numero-mensuel/2018-44/rmtc-volume-441-4-janvier-2018/plan-intervention-canada-face-menacesbiologiques.html

11. Organisation mondiale de la Santé. Rapport sur la santé dans le monde 2007 - Un avenir plus sûr: la sécurité sanitaire mondiale au XXle siècle. http://www.who.int/whr/2007/fr/ 